\title{
Improving treatment adherence among patients with chronic psychoses
}

\author{
Anjum Sair, Kamaldeep Bhui, Shujina Haq and Geraldine Strathdee
}

This paper presents the literature on compliance as an ideology and then examines strategles to improve patient outcomes if non-compliance is identified as undermining the treatment regimen. We present strategles used by our feam in a south London rehabilitation service for the severely mentally ill.

A thesaurus definition of 'compliance' is to 'submit, obey, surrender, conform, follow. ' Compliance' has also been defined as the extent to which a person's behaviour coincides with medical advice. Fawcett (1995) advocates a broadening of the behavioural context taken account of when patients accept (or not) a package of care. For example, attention to family, social and individualised interventions including education, problem-solving and cognitive-behavioural strategies have been advocated as part of the approach to maintain remissions. Fawcett (1995) recommended that the term 'adherence' be adopted (rather than compliance) as it places responsibility on the clinician to form a treatment alliance. This paper reviews the literature examining poor 'compliance' with medication and summarises the strategies advocated for the severely mentally ill.

Mental health is one of the most resource hungry areas of health care. The cost of treating schizophrenia consumes $1.6 \%$ of the total health care budget (Davies \& Drummond, 1994). Kane \& Borenstein (1985) suggested that non-compliance is more extensive in psychiatry as psychiatric illnesses have a significant effect on judgement, insight and stability. Treatment refusal among the severely mentally ill is argued to be a marker of poor insight, and to be one of the few predictive factors associated with violent behaviour and involuntary hospital admission. Various models within which compliance issues can be conceptualised are the biomedical, operant behavioural, health belief, educational and self-regulatory (Kemp \& David, 1995).

\section{Prevalence}

The reported incidence of non-compliance with antipsychotic medication ranges from $11-80 \%$.
Forty-eight per cent of patients are reported to be non-compliant within the first year of treatment, and $74 \%$ within the first two years (Weinden et al, 1986). Even during in-patient admissions up to $19 \%$ of psychiatric in-patients are not taking their drugs regularly, even though they are issued by nursing staff at appropriate times throughout the day. A survey of 253 psychiatric in-patients on the day of discharge reported that not more than half knew the name and dose of the medication, or why they were taking it, even though they had received group and individual medication instruction during their stay in hospital (Clary et al, 1992). Although 68\% knew the name of their psychiatric drugs, only $52 \%$ knew when to take them and $37 \%$ did not know why they were taking them.

\section{Causes of non-compliance}

Insight into the illness and the effects of medication

Younger, well-educated people with an affective illness rather than schizophrenia more often have adequate knowledge of their medication; deficits may therefore be specific to schizophrenia (Clary et al, 1992). Low IQ. cognitive impairment, lack of insight, grandiosity and medication-centred delusional beliefs have also been advocated as significant factors (Kemp \& David, 1995). Denial of illness is often assumed to be responsible for patients' refusal to comply. Yet patients can be designated to have no insight and still accept and derive benefits from medication. For some patients the adverse effects of medication can compound primary illness-related disability (Bebbington, 1995). Some patients fear that they can no longer distinguish their true personality from thoughts, feelings and behaviours that arise as a consequence of their medication.

\section{Communication between patients} and clinicians

Traditionally doctors have been poor at fully sharing information about medication with 
patients for fear of making the risks seem worse than the benefits. This notion in itself is presumptive and evidence in its own right of doctors having inadequate knowledge of their patients' views. Effective communication must therefore be at the heart of a collaborative approach. Patients are more likely to openly voice concerns about medications and objections rather than passively absent themselves from taking medication if they feel they would be heard and their concerns discussed 'fully' before a joint decision is made. Studies comparing psychiatrists' and patients' views about information regarding medication indicate a great mismatch. Warner et al (1994) surveyed psychiatrists and patients to elicit their views about essential medication related information. Sixteen per cent of patients used the physicians' desk reference as a source of information yet none of the psychiatrists believed this to be possible. Patients also regarded self-help groups as a useful source of information about side-effects, none of the psychiatrists believed this. Although a quarter of the patients stated that they never enquired about medication, none of the psychiatrists agreed that this was so. Furthermore, almost half of the patients indicated that they never asked to change their medication, while only $5 \%$ of psychiatrists believed this to be the case. Psychiatrists were also more likely than patients to attribute non-compliance to personality, to complex dosage schedules, or to misinformation about the medication that the patient had obtained. Seventy-three per cent of patients reported that they were compliant, if they thought that the medicine was efficacious, suggesting that patients were only likely to take their medication if they really believed it to be helpful, irrespective of the amount of information they received. Interpersonal skills have been reported to influence patient outcomes more than the quantity of patient instruction.

\section{Cognitive impairment and comorbidity}

Acute psychosis can lead to non-compliance because of the accompanying disorganisation, psychotic denial, negativism, grandiosity and premorbid personality traits (Kemp \& David, 1995). Up to $45 \%$ of the severely mentally ill also have a physical illness; frailty, poor memory and visual impairment consequently complicate treatment regimes and may compound nonadherence to the prescribed treatments.

\section{Instructions}

The European directive by the Council of Ministers placed an obligation upon pharmaceutical manufacturers to give more details on all newly-licensed medicines dispensed in an original pack and to include a user leaflet (George, 1994). For patients with multiple disabilities including disorders of perception and cognition, too much information may confuse further. The final objective of effective treatment episodes through a process of education may therefore be compromised.

\section{Dual diagnosis}

Substance misuse in dual diagnosis patients is not only associated with non-compliance with prescribed psychotropic medication, but may also make the prescribed psychotropic medication inadequate to maintain remission.

\section{Adverse effects}

The benefits of medication may only become apparent over several weeks but adverse effects are conspicuously present immediately. This may deter some patients from taking medication. especially if at first it results in very distressing adverse effects such as movement disorder (dystonias, extrapyramidal side-effects), sedation and dysphoria. Alternatively, any perceived benefit, if substantial, may outweigh the actual side-effects experienced but this evaluation is likely to be a dynamic one contextualised by other events in the patient's life. Some associated effects may lead to non-compliance because of the associated social disability. Thus neuroleptic associated obesity is common among those who default from treatment.

\section{Culture, self-determination and stigma}

Ethnic minorities appear to prefer lower doses and alternatives to medication (Bhui et al, 1995). Some patients will object to any mind altering drug on spiritual or religious grounds. Others may see drug treatment as another means of control by the dominant society with different cultural norms. There is some evidence that the pharmacokinetic and pharmacodynamic responses to medication differ in different cultural groups, and that there is a greater sensitivity to develop adverse effects among some minority ethnic populations (Lin et al, 1995). A careful collaborative effort to consider adverse effects and the cultural and attitudinal implications of psychotropic medication is essential. Stigma in ethnic minority communities will further compromise patients' ability to accept medication as a solution to their distress if they perceive this distress to be a response to socio-economic and interpersonal problems. 


\section{Strategies to improve collaboration}

The basis of most psychoeducational programmes is that it is collaboration rather than compliance between patients and therapist which will improve treatment adherence (Corrigan et al, 1990; Bebbington, 1995). Some programmes aim to improve knowledge about medication while others emphasise the practicalities of taking medication (Table 1). It is clear that such strategies need to be applied in the context of appropriate styles of consultation (Frank et al, 1995; Bebbington, 1995). Goldstein
(1995) has reviewed the range of family interventions which may give rise to better compliance. Integrating psychotic experience into normal life, accepting vulnerability to future episodes, dependence on psychotropics, establishing the significance of life events as stressors and distinguishing personality from disorder are the main components. Goldstein (1995) established the common elements of a variety of family approaches as being early engagement of the family, education about the disorder, communication, problem-solving and crisis intervention. Corrigan (1990) systematically outlined

Table 1. Psychoeducational interventions

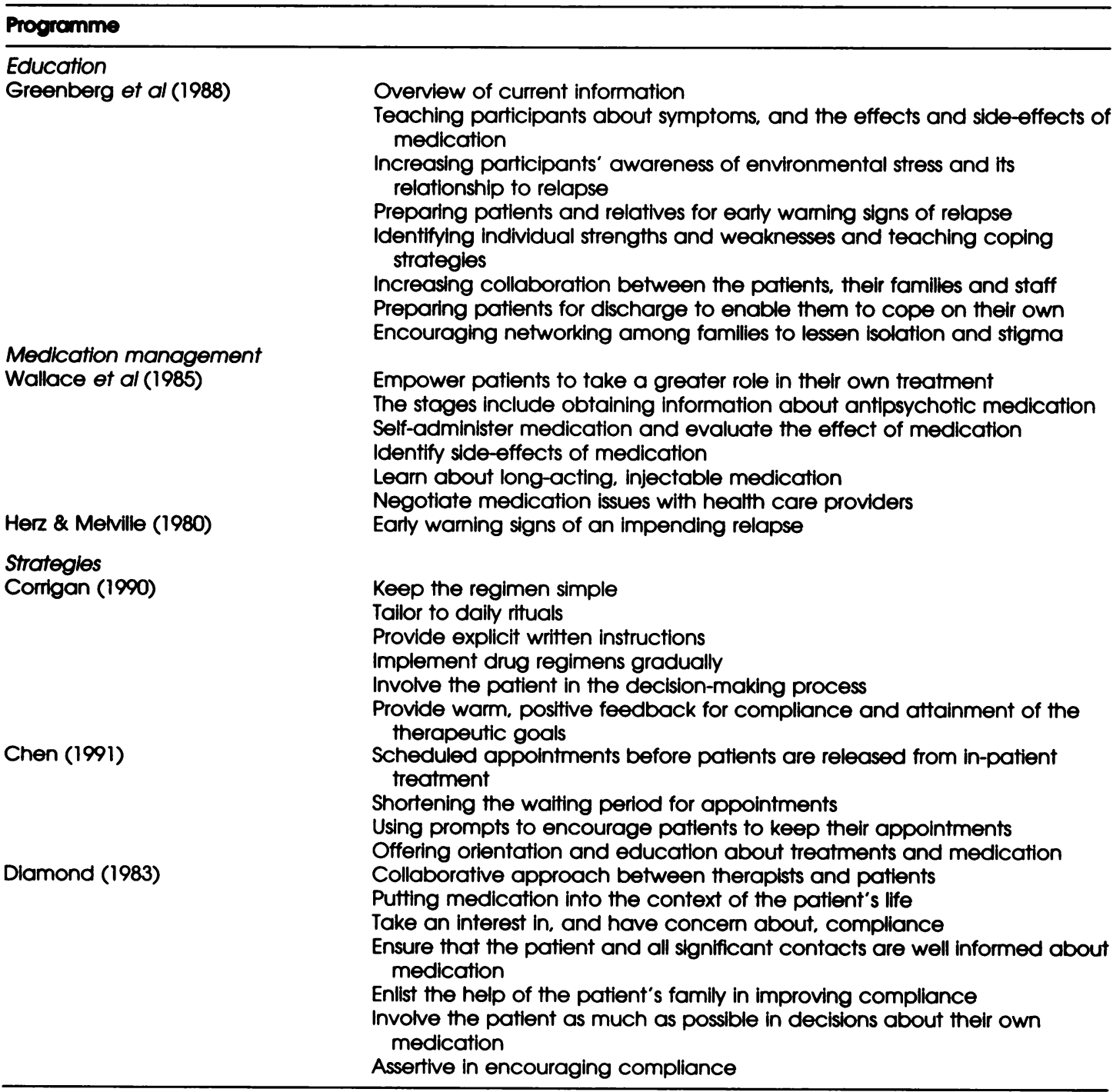


ORIGINAL PAPERS

Table 2. Barriers to collaboration (Corrigan et al, 1990)

\begin{tabular}{ll}
\hline Potential barriers & \\
\hline Medication related & $\begin{array}{l}\text { Side-effects } \\
\text { Complex treatment regimen } \\
\text { Long-term treatment } \\
\text { Cognitive disorganisation } \\
\text { lgnorance about illiness } \\
\text { Fatalistic attitude } \\
\text { Secondary gain from psychosis }\end{array}$ \\
Family related & $\begin{array}{l}\text { Family ignore benefits } \\
\text { Unrealistic family expectations } \\
\text { indifference }\end{array}$ \\
Clinician related & $\begin{array}{l}\text { Clinician believes patient has a poor prognosis } \\
\text { Aversive personal style of clinician } \\
\text { Clinician ignores patient's dissatisfaction } \\
\text { Aversive clinic setting } \\
\text { Lrganisational }\end{array}$ \\
& Lack of coordination in treatment delivery system \\
\hline
\end{tabular}

the potential barriers (Table 2) to a collaborative approach along with strategies to remedy each of these. Specific instrumental factors include structural and organisational elements of a service such as reasonable appointment times. clinic decor, the use of case managers and continuous treatment teams to properly coordinate care, and clinicians acquiring further training and counselling skills if their style of consultation is aversive.

\section{Influences on outcomes}

Many interventions have been offered on the basis of clinical experience but there are few randomised trials of specific interventions. Kemp \& David (1995) in a recent review cite only five studies as having a proper design. They conclude that behavioural approaches show benefit over didactic educational interventions, that education led to better attitudes and compliance and that cognitive interventions were effective. Kemp et als (1996) trial included 4-6 sessions of 20-60 minutes of 'compliance therapy'; this produced greater insight into the illness, better attitudes about drug treatment and greater compliance in the experimental compared with the control group. A number of studies indicate significant benefit to both patient and family following a psychoeducation programme, with clear evidence that medication was necessary but insufficient without family intervention and psychoeducation. Boczkowski et al (1985) investigated the effects of behavioural tailoring and a psychoeducation intervention on neuroleptic medication compliance. Behavioural tailoring involved identifying a highly visible location for placing medication or pairing medication intake with specific routine behaviour. Psychoeducational intervention was based on a standard educational approach. Pill count was taken as the criterion for compliance. Behavioural tailoring participants were significantly more compliant post-treatment than psychoeducation or control participants. Hence, behavioural techniques may be superior to a simple educational approach in improving compliance. Family treatment models which include a psychoeducational component as a major element of the programme also improve outcome (Leff et al, 1985). Anderson et al (1986) focused on improving the family's ability to manage the patient at home by providing basic information about the psychiatric disorder, symptoms, effects of medication and course of illness. In a recent study of elderly medical in-patients, the intervention group was encouraged to self-medicate (Lowe et al, 1995) and was found to have better compliance and knowledge scores at 10 days postdischarge. This finding has implications for how in-patients receive their medication given the trend towards decreasing length of in-patient admissions.

\section{Conclusions and implications}

The challenge to mental health professionals is to integrate these interventions into routine 
clinical practice. Training is essential yet the lack of identified funding for training packages, the absence of clinical guidelines specifically aimed at treatment adherence and the challenge of how to provide continuing professional development when it is skills-based learning that is necessary may undermine the implementation of these valuable techniques. An alliance between users and professionals emphasising to purchasers the importance of funding for such essential training is likely to bring about better implementation.

\section{References}

ANDERSON, C. M., REISS, D. J. \& HOGARTY, G. (1986) Schizophrenia and the Family. New York. Guilford.

BebBington, P. (1995) The content and context of compliance. International Clinical Psychopharmacology. 9 (suppl. 5). 41-50.

Bhu, K., Christe, Y. \& Bhugra, D. (1995) Essential elements of culturally sensitive psychiatric services. International Joumal of Social Psychiatry, 4, 242-256.

BOCZOWKSI, J. A., ZEICHNER, A. \& DESANTO, N. (1985) Neuroleptic compliance among chronic schizophrenic outpatients: an intervention outcome report. Joumal of Consulting and Clinical Psychology, 53, 666-671.

CHEN, A. (1991) Non compliance in community psychiatry: a review of clinical interventions. Hospital and Community Psychiatry. 42, 282-287.

Clary, C., Dever, A. \& Schweizer, E. (1992) Psychiatric inpatient's knowledge of medication at hospital discharge. Hospital and Community Psychiatry, 43. 140-144.

Corrigan, P. W., LIeberman, R. P. \& Engel, J. D. (1990) From non-compliance to collaboration in the treatment of schizophrenia. Hospital and Community Psychiatry. 41. 1203-1211.

DAVies, L. M. \& DRUMmond, M. F. (1994) Economics and schizophrenia: the real cost. British Journal of Psychiatry. 165 (suppl. 25), 18-21.

DiAMOND. R. J. (1983) Enhancing medication use in schizophrenic patients. Journal of Clinical Psychiatry. 44, 7-14.

FAWCETT, J. (1995) Compliance: definitions and key issues. Journal of Clinical Psychiatry. 66 (suppl. 1), 4-10.

FranK, E.. KUPFER, D. \& SiEgal, L. (1995) Alliance not compliance: a philosophy of outpatient care. Journal of Clinical Psychiatry. 66 (suppl. 1), 11-17.

GEORGE, C. F. (1994) What do patients need to know about prescribed drugs? Prescribers' Journal, 34, 7-11.
GoLDSTEIN, M. (1995) Psychoeducation and relapse prevention. International Clinical Psychopharmacology. 9 (suppl. 5), 59-69.

GREenBerG, L., Fine, S. B., LARSON, K., et al (1988) An inter-disciplinary psychoeducation program for schizophrenic patients and their families in an acute care setting. Hospital and Community Psychiatry. 39. 277-282.

HERZ, M. I. \& MELVILE, C. (1980) Relapse in schizophrenia. American Journal of Psychiatry, 137, 801-805.

KANE, J. M. \& BORENSTEIN, M. (1985) Compliance in long term treatment of schizophrenia. Psychopharmacology Bulletin, 21, 23-27.

KEMP, R. \& DAVID, A. (1995) Insight and adherence to treatment in psychotic disorders. British Journal of Hospital Medicine, 84, 222-227.

- HAYWARD. P., APPLEWHATtE. G.. et al (1996) Compliance therapy in psychotic patients: a randomised controlled trial. British Medical Journal, 312, 345-349.

LEFF, J., KUIPERS, L.. BERKOWITZ, R., et al (1985) A controlled trial of social intervention in the families of schizophrenic patients: a two year follow-up. British Journal of Psychiatry. 146, 594-600.

LiN, K., POLAND, R. \& ANDERSON, D. (1995) Psychopharmacology, ethnicity and culture. Transcultural Psychiatric Research Review. 32, 3-40.

LOWE, C.. RAYNOR, D. K., COURTNEY, E. R., et al (1995) Effects of self medication on knowledge of drugs and compliance with treatment in elderly patients. British Medical Journal, 310, 1229-1231.

WALLACE, C. J., BOONE. S. E., DONAHOE, C. P., et al (1985) Psychosocial rehabilitation for the chronic mentally disabled: social and independent living skills training. In Behaviour Treatment of Adult Disorders (ed. D. Barlow), pp. 462-501. New York: Guilford Press.

WARNER, L., Silk, K., YeATON, W. H., et al (1994) Psychiatrists' and patients' views on drug information sources and medication compliance. Hospital and Community Psychiatry. 45, 1235-1237.

WEINDEN, P. J., SHAw, E. \& MANN, J. J. (1986) Causes of neuroleptic non-compliance. Psychiatric Annals, 16. 571-575.

Anjum Sair, Clinical Pharmacist, *Kamaldeep Bhui, Senior Registrar, Maudsley Hospital; Shujina Haq; Medical Student, King's College School of Medicine and Dentistry, and Geraldine Strathdee, Consultant Psychiatrist, Maudsley Hospital, Denmark Hill, London SE5 8AZ

*Correspondence 